\title{
Magnetic field induced entropy change and magnetoelasticity in Ni-Mn-Ga alloys
}

\author{
Jordi Marcos, Antoni Planes, and Lluís Mañosa \\ Departament d'Estructura i Constituents de la Matèria, Facultat de Física, Universitat de Barcelona, Diagonal, 647, \\ E-08028 Barcelona, Catalonia, Spain \\ Fèlix Casanova, Xavier Batlle, and Amílcar Labarta \\ Departament de Física Fonamental, Facultat de Física, Universitat de Barcelona, Diagonal, 647, \\ E-08028 Barcelona, Catalonia, Spain \\ Benjamín Martínez \\ Institut de Ciència de Materials de Barcelona, CSIC, E-08193 Bellaterra, Catalonia, Spain \\ (Received 15 July 2002; published 18 December 2002)
}

\begin{abstract}
The magnetocaloric effect that originates from the martensitic transition in the ferromagnetic Ni-Mn-Ga shape-memory alloy is studied. We show that this effect is controlled by the magnetostructural coupling at both the martensitic variant and magnetic domain length scales. A large entropy change induced by moderate magnetic fields is obtained for alloys in which the magnetic moment of the two structural phases is not very different. We also show that this entropy change is not associated with the entropy difference between the martensitic and the parent phase — arising from the change in the crystallographic structure — which has been found to be independent of the magnetic field within this range of fields.
\end{abstract}

DOI: 10.1103/PhysRevB.66.224413

PACS number(s): 81.30.Kf, 75.80.+q, 75.30.Sg

\section{INTRODUCTION}

The physics of the magnetocaloric effect is an old subject that has been studied intensively in recent years. ${ }^{1}$ It is associated with the isothermal entropy change arising from the application of a magnetic field. The renewed interest in this subject arose after the discovery of materials which display a giant magnetocaloric effect as occurs, for instance, in systems simultaneously undergoing magnetic and structural phase transitions (magnetostructural phase transition). ${ }^{2-4} \mathrm{Be}-$ sides its evident technological importance for magnetic refrigeration applications, a proper understanding of the magnetocaloric effect is of great relevance in basic research. Among the systems that show a large magnetocaloric effect, an attractive candidate for study is the Ni-Mn-Ga Heusler alloy. ${ }^{5}$ This material has a twofold interest, as it displays both magnetocaloric and magnetic shape memory effects. The present paper deals with the physical origin of the magneticfield-induced entropy change related to the magnetocaloric effect, and also with the relationship between the magnetic shape-memory and magnetocaloric effects in this alloy.

The magnetic shape-memory property is a consequence of the coupling between structural and magnetic degrees of freedom in a ferromagnetic alloy undergoing a martensitic transition. ${ }^{6,7}$ The prototypical ferromagnetic shape-memory material is the Ni-Mn-Ga alloy, close to the stoichiometric Heusler composition, ${ }^{8,9}$ which transforms from an $L 2_{1}$ $(\mathrm{Fm} 3 \mathrm{~m})$ structure to a tetragonal martensitic phase. The coupling between magnetism and structure is assumed to occur at two well-separated length scales. At the microscopic scale spin-phonon coupling is considered to be weak as proven by the fact that very high fields are required to induce the transition even at temperatures very close to the transition temperature. Such weak coupling is also evidenced by the weak dependence of the phonon dispersion curves on an applied magnetic field. ${ }^{10}$ In contrast, at a mesoscale $(\sim 10 \mu \mathrm{m})$ corresponding to the martensitic variants (twin related) and magnetic domains length scales, the coupling ${ }^{11}$ is very strong, and large strains $(>5 \%)$ can be induced by the application of moderate fields. ${ }^{12}$ These strains are a consequence of the rearrangement of the twin related variants under the driving force originating from the difference in the Zeeman energy of the different variants. The conditions for such a rearrangement to occur are a strong (uniaxial) anisotropy of the tetragonal phase compared to that of the cubic phase, and a high mobility of the martensitic variants. Such a giant magnetostrictive behavior has attracted a great deal of interest since these materials are potential candidates for use as sensors and actuators. ${ }^{13}$

On the other hand, the magnetocaloric effect in the studied alloy arises from the magnetization jump accompanying the structural change. It has been argued ${ }^{14}$ that this behavior is similar to that observed in the $\mathrm{Gd}_{5}\left(\mathrm{Ge}_{x} \mathrm{Si}_{1-x}\right)_{4}$ (Ref. 2) and MnAs-based ${ }^{3,4}$ compounds, which also show a large magnetocaloric effect in the vicinity of a first-order magnetostructural transition. However, in those materials magnetic ordering from a paramagnetic to ferromagnetic phase occurs at the same time as the structural change. This is in contrast to Ni-Mn-Ga, which (in the composition range of interest) is ferromagnetic in both the parent and martensitic phases, and the magnetization jump is mainly controlled by the magnetostructural coupling at the mesoscopic scale. It is hence expected that both the magnetocaloric and shape-memory effects have the same physical origin. The present paper, based on experimental results, is aimed at providing evidence in support of this idea. Results also show that for moderated applied magnetic fields, the field-induced entropy is not related to the entropy change of the structural transition. The latter has been found to show a negligibly small dependence on the magnetic field. 


\section{RESULTS}

The studied sample was a single crystal, grown by the Bridgman technique, with a composition $\mathrm{Ni}_{49.5} \mathrm{Mn}_{25.4} \mathrm{Ga}_{25.1}$. Platelike specimens for calorimetry and magnetization measurements were prepared with the longer direction along the [100] and [110] crystallographic axes. The system exhibits an $L 2_{1}$ structure at room temperature, orders ferromagnetically at $T_{c} \simeq 381 \mathrm{~K}$, and undergoes a martensitic transition on cooling at $M_{S} \simeq 177 \mathrm{~K}$ to a modulated tetragonal structure $(5 R)$. On heating, the reverse transition takes place with a hysteresis of $\sim 10 \mathrm{~K}$.

Magnetization measurements were performed by the extraction technique in the range from 150 to $200 \mathrm{~K}$, under magnetic fields of up to $40 \mathrm{kOe}$. Calorimetric measurements under a magnetic field (0-50 kOe) were carried out using a high-sensitivity differential scanning calorimeter with a built-in magnetic field, especially designed for the study of magnetostructural transitions. ${ }^{15}$

Figure 1(a) shows the magnetization, $M$, versus temperature curves measured for the reverse transition on heating, for selected values of the applied field $H$. The magnetization displays a significantly abrupt change, $\Delta M$, at the martensitic transition. Interestingly, $\Delta M$ shows a strong dependence on $H$, which is illustrated in Fig. 1(b). $\Delta M \simeq 0$ for $H=0$, it reaches a minimum (negative) at a field $H^{*} \quad(=2$ $\pm 0.2 \mathrm{kOe})$, it vanishes at $H=H_{0}(=13 \pm 0.1 \mathrm{kOe})$, and for higher fields it saturates at a positive value. From the temperature dependence of the magnetization, the field-induced entropy change (as the field is increased from 0 to $H$ ) is obtained as

$$
\Delta S(0 \rightarrow H)=\int_{0}^{H}\left(\frac{\partial M}{\partial T}\right)_{H} d H
$$

The computed values of this entropy change are plotted in Fig. 2 as a function of $T$ for selected values of the magnetic field. It is worth noting that the obtained temperature dependence of $\Delta S(0 \rightarrow H)$ displays two peaks. This is a consequence of the fact that the martensitic transition takes place in two steps in the studied specimen [from Fig. 1(a) it can be seen that each step extends over a range of the order of $1 \mathrm{~K}$ ]. Discontinuous kinetics of this type is a typical characteristic of systems that undergo martensitic transitions caused by (unavoidable) small composition inhomogeneities, impurities, etc., which are known to slightly affect the actual transition path. ${ }^{16}$ Although the actual maximum value of the field-induced entropy change, $\Delta S_{\max }(H)$, is sensitive to the transition path in each specific sample, the field dependence of $\Delta S_{\text {max }}(H)$ (shown in the inset of Fig. 2) is not expected to depend on these features, since it is an intrinsic characteristic of the magnetostructural phase transition. Results show that this entropy change first increases with the field, reaches a maximum at $\sim 13 \mathrm{kOe}$, and decreases linearly with $H$ for higher fields. The maximum value of $\sim 0.65 \mathrm{~J} / \mathrm{K} \mathrm{mol}$ ( $\sim 11 \mathrm{~J} / \mathrm{K} \mathrm{kg}$ ) is comparable (except for the sign) to the absolute value reported for systems exhibiting a large magnetocaloric effect (resulting from a magnetostructural transi-
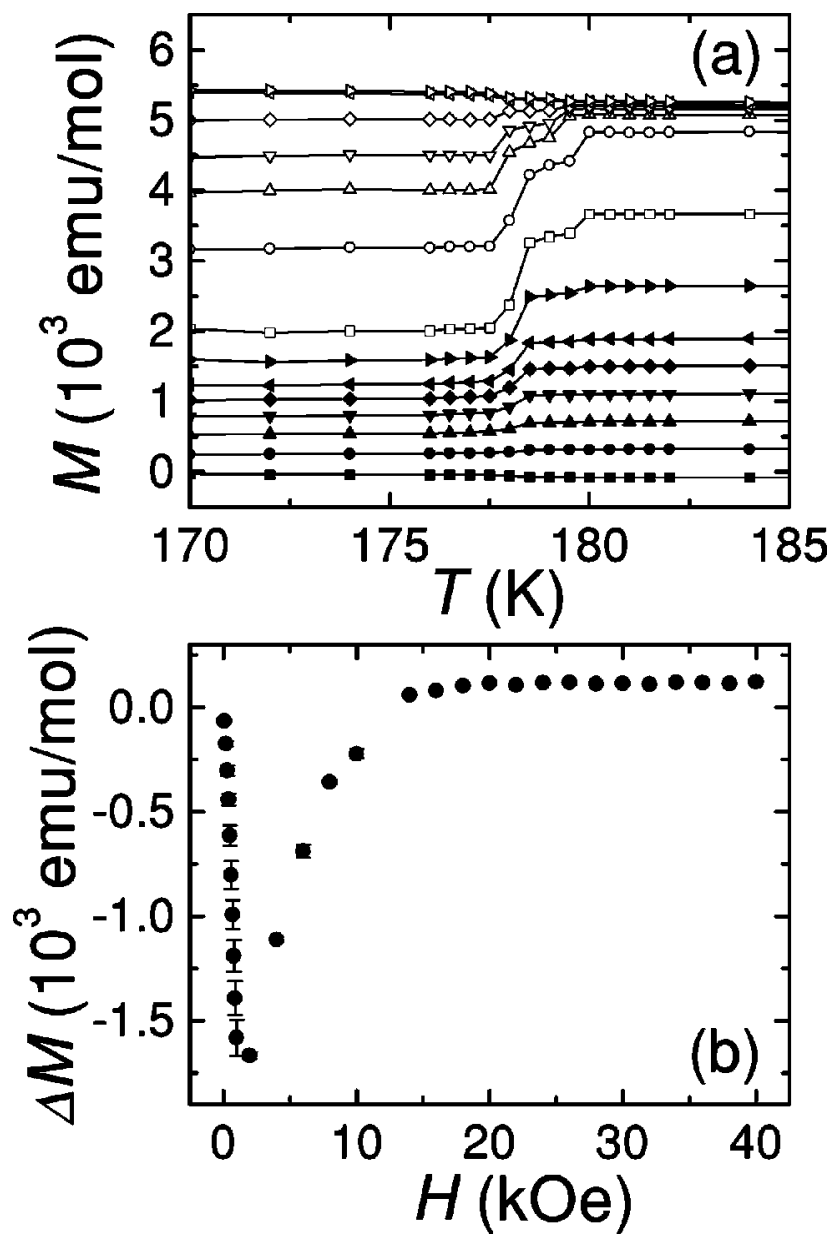

FIG. 1. (a) Magnetization vs temperature at selected values of the magnetic field applied along the [100] direction of the cubic phase. Measurements were performed during heating. The different symbols correspond to the following fields: $H=0$ (ם), $100 \mathrm{Oe}$ $(\boldsymbol{\bullet}), 200 \mathrm{Oe}(\boldsymbol{\Delta}), 300 \mathrm{Oe}(\boldsymbol{\nabla}), 400 \mathrm{Oe}(\bullet), 500 \mathrm{Oe}(\boldsymbol{\bullet}), 700 \mathrm{Oe}$ $(\triangleright), 1 \mathrm{kOe}(\square), 2 \mathrm{kOe}(\bigcirc), 4 \mathrm{kOe}(\triangle), 6 \mathrm{kOe}(\nabla), 10 \mathrm{kOe}$ $(\diamond), 20 \mathrm{kOe}(\triangleleft)$ and $40 \mathrm{kOe}(\triangleright)$. (b) Magnetization change at the phase transition, as a function of the magnetic field.

tion) such as the $\mathrm{Gd}_{5} \mathrm{Ge}_{2} \mathrm{Si}_{2}(\sim 14 \mathrm{~J} / \mathrm{K} \mathrm{kg}$ at $H=20 \mathrm{kOe})$ and the $\mathrm{MnFeP}_{0.45} \mathrm{As}_{0.55}$ compounds $(\sim 15 \mathrm{~J} / \mathrm{K} \mathrm{kg}$ at $H$ $=20 \mathrm{kOe}){ }^{4}$

In order to compare the entropy change induced by the application of a magnetic field with the entropy change associated with the crystallographic change (transition entropy change), we have carried out calorimetric measurements under a magnetic field. Examples of the recorded thermograms at selected values of $H$ are shown in Fig. 3. From the recorded heat flux $d q / d t$ and temperature $T(t),{ }^{17}$ and after a proper correction of the base line, the entropy change associated with the phase transition is obtained as

$$
\Delta S=\int_{T_{i}}^{T_{f}} \frac{1}{T} \frac{d q}{d T} d T,
$$

where $T_{i}$ and $T_{f}$ are temperatures located, respectively, above (below) and below (above) the starting and finishing temperatures on cooling (heating). For fields ranging from 0 


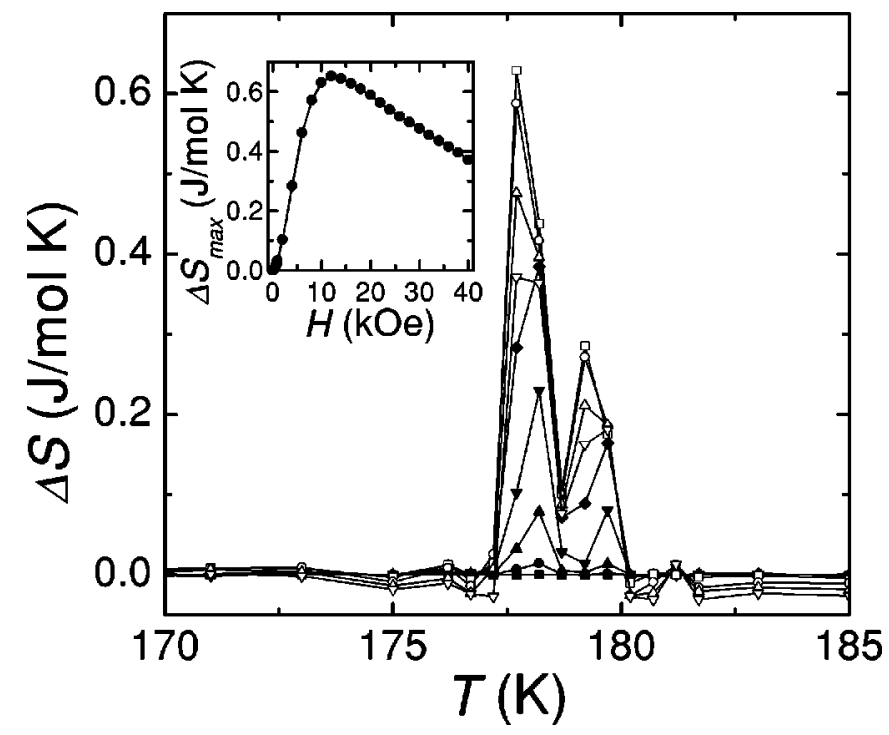

FIG. 2. Field-induced entropy change as a function of temperature for selected values of the maximum applied field. The correspondence between symbols and measured fields is the following: $H=100$ Oe $(\boldsymbol{\square}), 500$ Oe $(\boldsymbol{O}), 1 \mathrm{kOe}(\boldsymbol{\Delta}), 2 \mathrm{kOe}(\boldsymbol{\nabla}), 4 \mathrm{kOe}$ $(\diamond), 10 \mathrm{kOe}(\square), 20 \mathrm{kOe}(\bigcirc), 30 \mathrm{kOe}(\triangle)$ and $40 \mathrm{kOe}(\nabla)$. The inset shows the maximum value of the field-induced entropy change as a function of the magnetic field.

to $50 \mathrm{kOe}$, the transition entropy change has been found to remain constant within the experimental errors. An average value $\Delta S=-0.55 \pm 0.03 \mathrm{~J} / \mathrm{Kmol}$ has been obtained, which agrees well with the reported values for a $L 22_{1}$ to $5 R$ transition in Ni-Mn-Ga. ${ }^{18,19}$

It is also possible to obtain the transition hysteresis loops (martesitic transformed fraction, $y$, versus $T$ ) from calorimetric data. The martensitic transformed fraction is obtained as $y=\Delta S(T) / \Delta S$ for the forward transition on cooling, and $y$

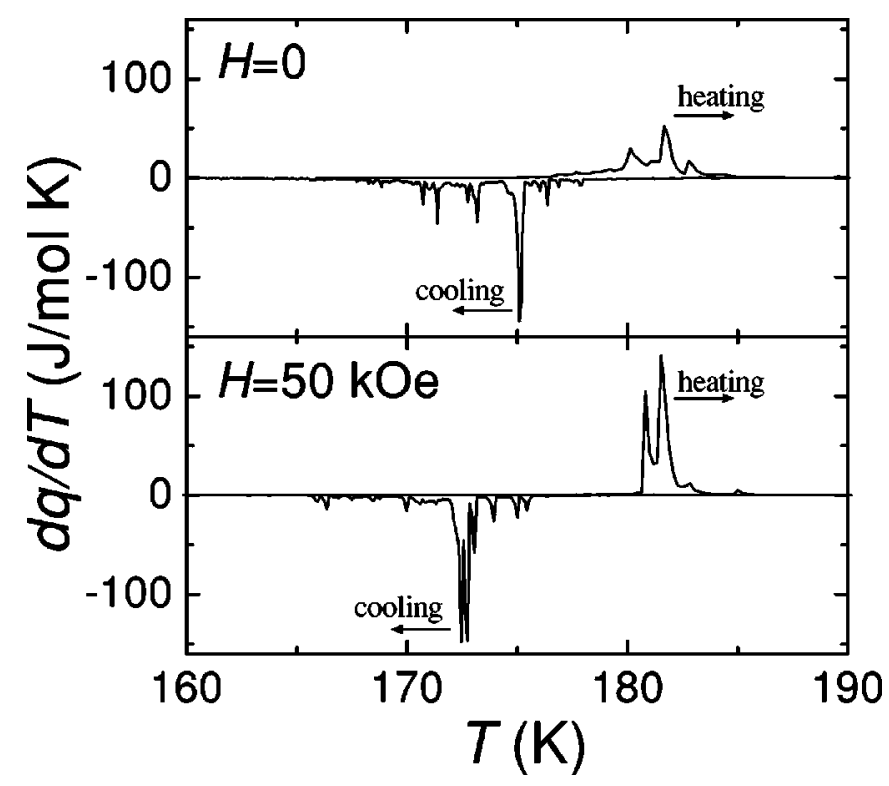

FIG. 3. Thermal curves corresponding to the forward (cooling) and reverse (heating) martensitic transitions at selected values of the applied magnetic field.

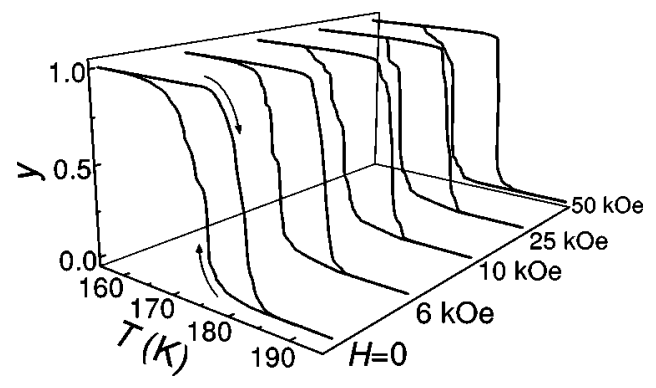

FIG. 4. Transformed fractions $y=\Delta S / \Delta S_{\max }$ as a function of temperature for forward and reverse transitions (hystheresis loops), computed from calorimetric data, at different values of the magnetic field.

$=1-\Delta S(T) / \Delta S$ for the reverse transition on heating, with $\Delta S(T)=\int_{T_{i}}^{T}(d q / d T) d T / T\left(T<T_{i}\right.$ on cooling and $T>T_{i}$ on heating). The obtained hysteresis loops are shown in Fig. 4, for the different applied fields. On increasing the magnetic field, the loop becomes sharper and broader.

\section{DISCUSSION}

In order to account for the field dependence of $\Delta S_{\max }(H)$ we propose a description of the transition mechanism under an applied magnetic field based upon the experimental observations of the magnetic and structural domain patterns formed at different applied fields, ${ }^{20,21}$ which is aimed at describing the nature of magnetostructural coupling at the mesoscale level. When the transition takes place at zero field, the nucleation gives rise to plates formed by parallel strips of twin-related variants. The appropriate amount of each variant $(v=1$ and 2$)$ ensures that parent-martensite interfaces satisfy the invariant plane strain condition ${ }^{22}$. It is worth noting that the nucleation of plates with such a mesostructure is very advantageous since it minimizes the elastic (strain) energy arising from the crystal lattice misfit along the interfacial boundaries. $^{22,23}$ Magnetic domains are formed within each variant in such a way that the magnetization alternates between two values $\pm \mathbf{M}_{M(v)}$ along the corresponding easy axis ( $c$ axis of the tetragonal structure), where the subscript $M$ refers to martensite. This configuration ensures the absence of "magnetic charges." In contrast, if the sample is cooled through the transition under a magnetic field larger than the saturating field of the high temperature phase $\left(>H^{*}\right)$, the twin related variants are magnetized. As the field is increased, due to the strong uniaxial anisotropy of the tetragonal phase, the Zeeman energy difference $\left(\left[\mathbf{M}_{M(1)}-\mathbf{M}_{M(2)}\right]\right.$ - H) between neighboring variants is minimized by increasing the fraction of those variants with their easymagnetization axis forming a smaller angle with the applied field. Finally, transformation under high enough fields $\left(>H_{0}\right)$ results in a magnetically saturated single variant crystal. ${ }^{24}$ As described above and assuming that the magnetic anisotropy of the high-temperature phase is weak, the magnetization difference between the two phases, for $H>H^{*}$, can be expressed as $\Delta M=\left\{\left[f \mathbf{M}_{M(1)}+(1-f) \mathbf{M}_{M(2)}\right]-\mathbf{M}_{P}\right\}$ $\cdot \hat{\mathbf{h}}$, where $f=f(\mathbf{H})$ is the fraction of variants favored by 
application of the magnetic field, which is a function of $\mathbf{H} ; \hat{\mathbf{h}}$ is a unit vector along the direction of the field, and $\mathbf{M}_{P}$ is the magnetization of the parent phase. The magnetization along the direction of the applied magnetic field is thus supposed to be of the form $M(T, H)=M_{P}(H)+\Delta M F([T$ $\left.\left.-T_{t}(H)\right] / \Delta T\right)$. In the preceding expression the magnetization of the parent phase, $M_{P}(H)$, is assumed to be temperature independent, and $F\left(\left[T-T_{t}(H)\right] / \Delta T\right)$ is a monotonically decreasing function of width $\Delta T$ (independent of $H$ ) which accounts for the change in magnetization within the transition region, such that $F \rightarrow 0$ for $T \gg T_{t}(H)$ and $F \rightarrow 1$ for $T$ $\ll T_{t}(H)$ (for $\Delta T \rightarrow 0, F$ is the Heaviside function). $\Delta T$ is a measure of the temperature range over which the transition spreads. By using Eq. (1), the maximum value of fieldinduced entropy change can be calculated as

$$
\Delta S_{\max }(H) \cong-\frac{m_{M} V}{\Delta T} \int_{0}^{H} f(H) d H+\frac{m_{P} V}{\Delta T} H,
$$

where it has been assumed that for moderated fields, the transition is very little shifted under the application of $H$ and that $\partial F / \partial T \simeq-1 / \Delta T$ is constant. In Eq. (3), $m_{P}$ is the magnetic moment of the parent phase (saturation value) and $m_{M}=\left(m_{M(1)}+m_{M(2)}\right)$ is approximately the magnetic moment in the martensitic phase. $m_{M(1)}$ and $m_{M(2)}$ are respectively the projections of the magnetic moments of the martensitic variants [(1) and (2)] along the direction of the field (notice that $\mathbf{M}_{M(1)}$ and $\mathbf{M}_{M(2)}$ form an angle very close to $\pi / 2$ ). This result shows that, for small fields, the magnetocaloric effect mainly arises from the mesoscale magnetostructural coupling [accounted for by the function $f(H)$ ]. In order to proceed further, it is necessary to assume a form for the function $f(H)$. Micromagnetic models may provide specific forms for this function. ${ }^{6,7}$ Here, based on experimental results, we assume that the rate of change of $f$ with $H$ is proportional to $-f$. Therefore, the following phenomenological expression is proposed for fields larger than $H^{*}$ : $f(x)=1-\frac{1}{2} e^{-x}$, where $x=H / H_{c}\left[f(x) \rightarrow \frac{1}{2}\right.$ for $x \rightarrow 0$, and $f(x) \rightarrow 1$ for $x \rightarrow \infty$ ]. $H_{c}$ is the characteristic field of the exponential function, which is close to $H^{*}$. This simple function is suitable for describing the field dependence of the fraction of favored variants in a mean-field sense. The corresponding expressions for $\Delta M$ and $\Delta S_{\max }$ read

$$
\Delta M(x) \simeq V \Delta m\left[1-\frac{m_{M}}{2 \Delta m} e^{-x}\right]
$$

and

$$
\Delta S_{\max }(x) \simeq-\frac{V \Delta m H_{c}}{\Delta T}\left[x+\frac{m_{M}}{2 \Delta m}\left(e^{-x}-1\right)\right],
$$

where $\Delta m=m_{M}-m_{P}$ is the change in magnetic moment between the martensitic and parent phases. In Fig. 5 the scaled quantities $\Delta M / V \Delta m$, and $\Delta S_{\max }(H) \Delta T / V H_{c} \Delta m$, given by Eqs. (4) and (5) are plotted (lines) as a function of $x$ for different values of the ratio $\Delta m / m_{M}$. The behavior can be compared to the experimental data. In this case, the value of $\Delta m / m_{M}$ can be obtained from the magnetization curves and, therefore, it is no longer a free fitting parameter. For the alloy

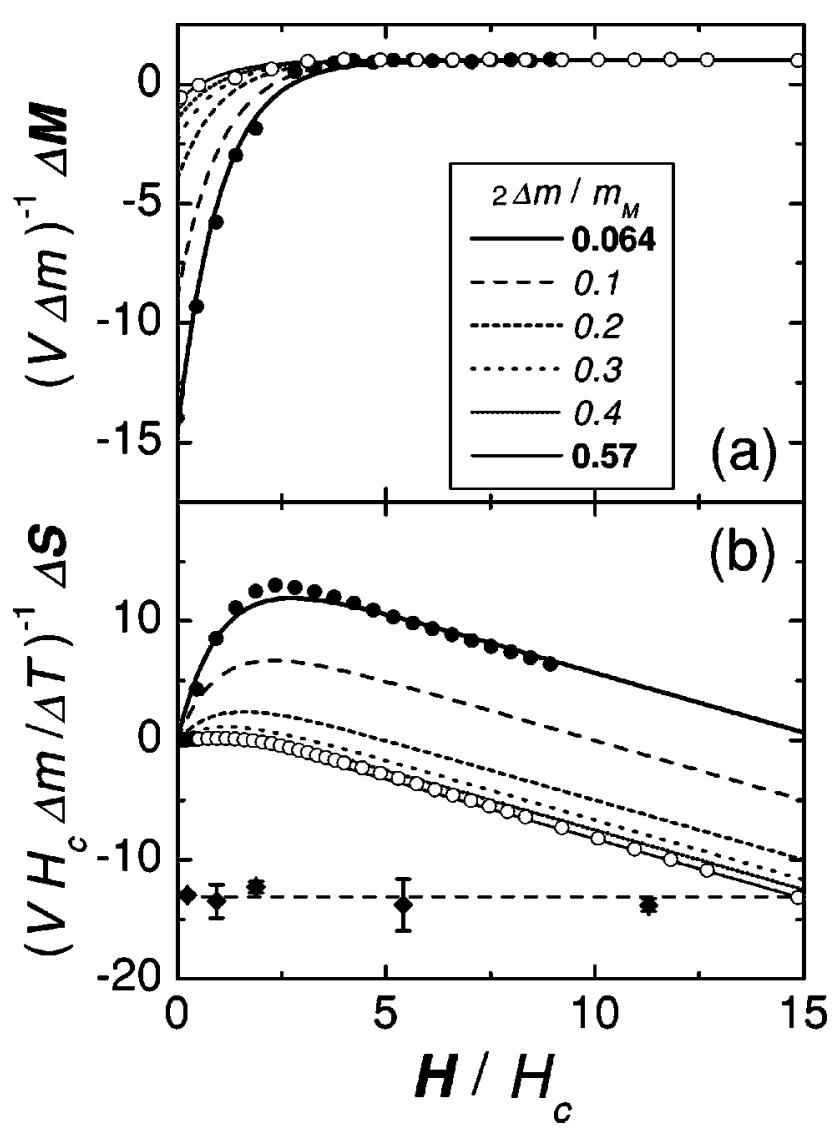

FIG. 5. (a) Normalized change in the magnetization as function of $x\left(=H / H_{c}\right)$ for different values of $\Delta m / m_{M}$. (b) Maximum value of the field-induced entropy for different values of $\Delta m / m_{M}$. Lines correspond to calculated values, and symbols to experimental data for the present sample (,$H_{c}=4250 \mathrm{Oe}$ ), and for sample in Ref. $14\left(\bigcirc, H_{c}=2300 \mathrm{Oe}\right)$. Calorimetric data for the transition entropy ( ) are also shown (in this case the line is just a guide to the eyes).

studied in the present work, $2 \Delta m / m_{M}=0.064$ and for a NiMn-Ga single crystal sample with $23.1 \% \mathrm{Mn}$ and $24.3 \%$ $\mathrm{Ga},{ }^{14} 2 \Delta m / m_{M}=0.57$. The agreement between experimental data and the calculated curves is remarkable. In order to compare the field-induced entropy change with the transition entropy change, we have also included the values measured calorimetrically (conveniently renormalized) in the figure as a function of $x\left(=H / H_{c}\right)$. The evident independence of these values on the magnetic field shows that the field-induced entropy and the transition entropy changes are indeed independent quantities. Actually, this finding is in agreement with recent first-principle calculations which indicate that the transition from cubic to tetragonal structures is driven by the vibrational free energy. ${ }^{25}$

Let us now discuss the effect of the magnetic field on the hysteresis loops (see Fig. 4). As already mentioned, two interesting features show up as the field is increased: (i) the transition becomes sharper, and (ii) the area of the loops increases. Both effects are a direct consequence of the magnetostructural coupling. Actually, the area $A(H)=\oint y d T$ of the loops yields the dissipated energy per cycle, $E_{\text {diss }}$ $\simeq A(H) \Delta S,{ }^{26}$ which is related to the nucleation barriers. It is found that such dissipated energy is a function of the mag- 


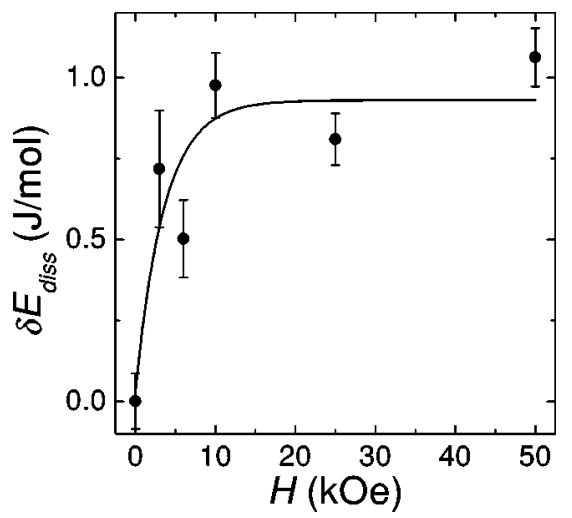

FIG. 6. Difference in the dissipated energy $\left[\delta E_{d i s s}=E_{\text {diss }}(H)\right.$ $\left.-E_{d i s s}(0)\right]$ as a function of the applied magnetic field.

netic field. The difference in dissipated energy $\delta E_{\text {diss }}$ $=E_{\text {diss }}(H)-E_{\text {diss }}(0)$, as a function of $H$, is shown in Fig. 6 . The results shown correspond to the magnetic field applied along the [110] axis. Results are similar when the field is applied along the [100] axis. Nevertheless, it has been found that the dissipated energy is sensitive to the previous magnetothermal history of the material. The procedure we have followed is: (i) annealing above the Curie point, (ii) zerofield cooling, and (iii) temperature cycling across the martensitic transition. Each transformation cycle was performed at a constant magnetic field larger than that of the previous cycle. The observed increase of $\delta E_{\text {diss }}$ with the magnetic field can be explained by taking into account the tendency of the system to nucleate a single variant martensite as the magnetic field is increased. This is a consequence of the fact that the application of a magnetic field breaks the structural and magnetic symmetry. The breakdown of this symmetry also explains the fact that the transition becomes sharper: the formation of a single variant martensite enhances, as explained above, the elastic strain energy associated with the parentmartensite interface matching. This increase of elastic energy may be compensated for by the corresponding decrease in the Zeeman energy. This suggests that in the range from $H^{*}$ to $\sim H_{0}, \delta E_{\text {diss }}$ is proportional to the magnetic energy $\mathbf{H}$ $\cdot \Delta \mathbf{M}$. Our experimental results (see Fig. 6) are in agreement with such an interpretation.

The measured values of the transition entropy change and magnetization change (at saturation) enable us to estimate the shift in the equilibrium transition temperature $\left(T_{0}\right)$ caused by the magnetic field, by using the ClausiusClapeyron equation:

$$
\frac{d T_{0}}{d H}=-\frac{\Delta M_{\text {sat }}}{\Delta S} .
$$

Taking $\Delta M_{\text {sat }}=120 \pm 10 \mathrm{emu} / \mathrm{mol}$ and $\Delta S=-0.55$ $\pm 0.03 \mathrm{~J} / \mathrm{K} \mathrm{mol}, d T_{0} / d H=22 \pm 3 \mathrm{mK} / \mathrm{kOe}$. Notice that this finding is in contrast to what occurs in $\mathrm{Gd}_{5}\left(\mathrm{Ge}_{x} \mathrm{Si}_{1-x}\right)_{4}$ and $\mathrm{MnAs}_{1-x} \mathrm{Sb}_{x},{ }^{2,3}$ for which there is a significant shift of the transition temperature with the applied field $\left(d T_{t} / d H\right.$ $\sim 400 \mathrm{mK} / \mathrm{kOe}$ ).

For thermoelastic martensitic transitions, the transition temperature is usually estimated as $T_{0}=\left(M_{s}+A_{f}\right) / 2$, where
$M_{s}$ is the martensite starting temperature and $A_{f}$ the finishing temperature of the reverse transition. By using the calorimetric data to compute $T_{0}$ at different values of the magnetic fields we obtain: $d T_{0} / d H=-30 \pm 8 \mathrm{mK} / \mathrm{kOe}$. The apparent inconsistency between this value and the value obtained from Clausius-Clapeyron equation originates from the fact that the estimation of the equilibrium temperature is strongly influenced by nucleation effects which do not act symmetrically on forward and reverse transitions. Actually, this may explain why some authors have reported a magnetic-field decrease of the transition temperature, ${ }^{8}$ while others have mentioned an increase,${ }^{27}$ and others no magnetic-field dependence at all. ${ }^{28}$

\section{SUMMARY AND CONCLUSIONS}

The magnetocaloric effect in Ni-Mn-Ga alloys has been studied by magnetization measurements and calorimetric measurements under a magnetic field. A model has also been proposed to account for this effect. Although simple, this model contains the essential physical ingredients to characterize the magnetocaloric effect in the vicinity of a first-order transition. In particular, it reproduces fairly well the experimental results presented here as well as those reported in Ref. 14.

It has been shown that the magnetocaloric effect in $\mathrm{Ni}$ $\mathrm{Mn}-\mathrm{Ga}$ alloys is directly related to the magnetostructural coupling taking place at the length scale of the magnetic and martensitic domains. The fraction $f(\mathbf{H})$ of martensitic variants with the easy magnetic axis best suited for the applied magnetic field is the relevant quantity to be considered. A phenomenological function $f$ has been proposed which is adequate to reproduce the experimental observations. It is argued that this function is also relevant in describing the magnetic shape-memory effect since it has the same physical origin as the magnetocaloric effect in the Ni-Mn-Ga alloy: twin related variants rearrangement in the martensitic phase under the application of a magnetic field. Actually, the coupling between magnetism and strain is commonly expressed in terms of a piezomagnetic coefficient, $p_{12}=d \epsilon / d H$. Within the framework used here to analyze the magnetocaloric effect, for $H>H^{*}$, the effective strain generated by the application of a magnetic field is given by $\epsilon^{e f f}=f(\mathbf{H}) \epsilon_{1}+[1$ $-f(\mathbf{H})] \epsilon_{2}$, where $\epsilon_{1}$ and $\epsilon_{2}$ are the components of the strain tensor giving rise to the deformation (with respect to the cubic phase) of variants (1) and (2), respectively. The resulting piezomagnetic coefficient reads $p_{12}=\Delta \epsilon d f / d H$.

The specific features of the magnetocaloric effect in this system have been found to be controlled by the difference in the magnetic moment between the martensite and the hightemperature phase $\left(\Delta m / m_{M}\right)$. When this quantity is small, the field-induced entropy change is first positive, reaches a maximum for a field close to the field necessary to induce a single variant martensite, and decreases linearly with $H$ for higher fields. For high values of $\Delta m / m_{M}$, only the linearly decreasing region is observed. The physical mechanism governing the magnetocaloric effect in the Ni-Mn-Ga alloy has been found to be very different from that in the $\mathrm{Gd}_{5}\left(\mathrm{Ge}_{x} \mathrm{Si}_{1-x}\right)_{4}$ or MnAs-based compounds, for which this 
effect also originates from a magnetostructural transition. Although in these systems the magnetocaloric effect is also very large in the vicinity of the transition, the field-induced entropy change mainly results from the transition entropy change (due to the high value of the change of transition temperature with $H)^{29}$, while in Ni-Mn-Ga the weak magnetostructural coupling at a microscopic level results in a transition entropy change independent of the magnetic field.

\section{ACKNOWLEDGMENTS}

We thank A. Saxena for a critical reading of the manuscript. This work received financial support from CICyT (Project Nos. MAT2001-3251 and MAT2000-0858) and DURSI (Project No. 2001SGR00066). J.M. and F.C. acknowledge financial support from DURSI.
${ }^{1}$ A. M. Tishin, in Handbook of Magnetic Materials, edited by K. H. J. Buschow, (North-Holland, Amsterdam, 1999), Vol. 12, Chap. 4, pp. 3950-524.

${ }^{2}$ V. K. Pecharsky and K. A. Gschneider, Jr., Phys. Rev. Lett. 78, 4494 (1997).

${ }^{3}$ H. Wada and Y. Tanabe, Appl. Phys. Lett. 79, 3302 (2001).

${ }^{4}$ O. Tegus, E. Brück, K. H. J. Buschow, and F. R. de Boer, Nature (London) 415, 450 (2002).

${ }^{5}$ F. Hu, B. Shen, and J. Sun, Appl. Phys. Lett. 76, 3460 (2000).

${ }^{6}$ R. C. O’Handley, J. Appl. Phys. 83, 3263 (1998).

${ }^{7}$ R. D. James and M. Wuttig, Philos. Mag. A 77, 1273 (1998).

${ }^{8}$ K. Ullako, J. K. Huang, C. Kantner, R. C. O'Handley, and V. V. Kokorin, Appl. Phys. Lett. 69, 1966 (1996).

${ }^{9}$ A. Planes and Ll. Mañosa, Solid State Phys. 55, 159 (2001).

${ }^{10}$ Ll. Mañosa, A. Planes, J. Zarestky, T. Lograsso, D. L. Schlagel, and C. Stassis, Phys. Rev. B 64, 024305 (2001).

${ }^{11}$ The coupling at mesoscale will be referred as magnetostructural coupling in order to distinguish it from conventional magnetoelastic coupling, which is responsible for standard magnetostriction.

${ }^{12}$ S. J. Murray, M. Marioni, S. M. Allen, and R. C. O'Handley, Appl. Phys. Lett. 77, 886 (2000).

${ }^{13}$ T. Kakeshita and K. Ullakko, MRS Bull. 27, 105 (2002).

${ }^{14}$ F. Hu, B. Shen, J. Sun, and G. Wu, Phys. Rev. B 64, 132412 (2001).

${ }^{15}$ J. Marcos, F. Casanova, X. Batlle, A. Labarta, A. Planes and Ll. Mañosa (unpublished).
${ }^{16}$ F. C. Lovey and V. Torra, Prog. Mater. Sci. 44, 189 (1999).

${ }^{17}$ The heat flux and temperature were simultaneously recorded at a rate of $0.25 \mathrm{~Hz}$.

${ }^{18}$ V. A. Chernenko, E. Cesari, V. V. Kokorin, and I. N. Vitenko, Scr. Metall. Mater. 33, 1239 (1995).

${ }^{19}$ A. Planes, E. Obradó, A. Gonzàlez-Comas, and L1. Mañosa, Phys. Rev. Lett. 79, 3926 (1997).

${ }^{20}$ Q. Pan and R. D. James, J. Appl. Phys. 87, 4702 (2000).

${ }^{21}$ H. D. Chopra, C. Ji, and V. V. Kokorin, Phys. Rev. B 61, R14 913 (2000).

${ }^{22}$ A. G. Khachaturyan and G. A. Shatalov, Zh. Eksp. Teor. Fiz. 56, 1037 (1969)] [Sov. Phys. JETP 29, 557 (1969)]; A. G. Khachaturyan, S. M. Shapiro and S. Semenovskaya, Phys. Rev. B 43, 10832 (1991).

${ }^{23}$ G. R. Barsch, B. Horovitz, and J. A. Krumhansl, Phys. Rev. Lett. 59, 1251 (1987).

${ }^{24}$ The rotation of magnetization within each martensitic variant is neglected since the martensitic phase has a strong magnetic anisotropy.

${ }^{25}$ J. Enkovaara, A. Ayuela, L. Nordström, and R. M. Nieminen, J. Appl. Phys. 91, 7798 (2002).

${ }^{26}$ J. Ortín and A. Planes, Acta Metall. 37, 1433 (1989).

${ }^{27}$ Y. Ma, S. Awaji, K. Watanabe, M. Matsumoto, and N. Kobayashi, Appl. Phys. Lett. 76, 37 (2000).

${ }^{28}$ F. Zuo, X. Su, and K. H. Wu, Phys. Rev. B 58, 11127 (1998).

${ }^{29}$ F. Casanova, X. Batlle, A. Labarta, J. Marcos, Ll. Mañosa, and A. Planes, Phys. Rev. B 66, 100401(R) (2002). 